\title{
On expansions in neutrino effective field theory
}

\section{Gitte Elgaard-Clausen and Michael Trott}

Niels Bohr International Academy and Discovery Centre, Niels Bohr Institute,

University of Copenhagen,

Blegdamsvej 17, DK-2100 Copenhagen, Denmark

E-mail: gitteec@gmail.com, michael.trott@cern.ch

ABSTRACT: We match the seesaw model for generating neutrino masses onto the Standard Model Effective Field Theory (SMEFT). We perform this matching at tree level up to dimension seven in the operator expansion. We explain how some of the perturbations of the neutrino mass matrix due to operators of mass dimension greater than five are tied to integrating out the heavy Majorana mass eigenstates in sequence. We demonstrate that the low energy limit of seesaw models are well described by the SMEFT, particularly when constructed using a flavour space expansion. Flavour space expansions of seesaw models are of interest as the coupling of the heavy states to the Standard Model, that are integrated out to generate neutrino masses, are through flavour space vectors $\in \mathbb{C}^{3}$. We point out that neutrino phenomenology can be systematically developed as a perturbation around the unknown eigenvectors diagonalizing the charged lepton mass matrix using the fact that these eigenvectors also form a basis of $\mathbb{C}^{3}$. This point holds in seesaw models and can also be applied to other models of neutrino mass generation to develop systematic expansions. We develop the algebra for this flavour space and discuss some phenomenology to illustrate this approach.

Keywords: Effective Field Theories, Neutrino Physics

ARXIV EPRINT: 1703.04415 


\section{Contents}

1 Introduction 1

2 Full theory for a minimal seesaw scenario 3

2.1 Equations of motion of the seesaw theory 4

3 Matching the seesaw to the SMEFT 4

$3.1 \mathcal{L}^{(5)}$ matching 5

$3.2 \mathcal{L}^{(6)}$ matching $\quad 6$

$\begin{array}{lll}3.3 & \mathcal{L}^{(7)} \text { matching } & 7\end{array}$

4 Perturbation and non-perturbation of the neutrino mass matrix $\quad 8$

$\begin{array}{lll}4.1 \text { Time ordered products of } \mathcal{L}^{(6)} \text { and } \mathcal{L}^{(4)} & 10\end{array}$

$\begin{array}{ll}4.2 \text { The neutrino mass matrix up to } \mathcal{L}^{(7)} & 11\end{array}$

5 Flavour space expansion for the eeesaw 11

$\begin{array}{ll}5.1 \text { Developing the FSE } & 12\end{array}$

6 Conclusions 15

$\begin{array}{lll}\text { A } & \mathbb{C}^{3} \text { algebra for eigenvectors diagonalizing a mass matrix } & 16\end{array}$

$\begin{array}{ll}\text { B Operator basis of ref. [1] } & 18\end{array}$

\section{Introduction}

Recently, there has been an escalation of theoretical efforts treating the Standard Model (SM) as a consistent low energy limit of a more fundamental theory. This is a natural result of the discovery of a dominantly $J^{P}=0^{+}$Higgs like boson at the Large Hadron Collider (LHC), and increased experimental indications that there is a mass gap between the electroweak scale $(v \sim 246 \mathrm{GeV})$ and any scale of new physics. It is reasonable to assume that the SM Lagrangian terms are the leading terms in the Standard Model Effective Field Theory (SMEFT) operator expansion [1-9].

Despite the power of the SMEFT formalism and recent systematic developments, there is little direct experimental evidence that higher dimensional operators supplementing the SM have non-vanishing Wilson coefficients. One exception is arguably supplied by the Wilson coefficient of the dimension five operator given by $[2,3]$,

$$
\mathcal{Q}_{5}^{\beta \kappa}=\left(\overline{\ell_{L}^{c, \beta}} \tilde{H}^{\star}\right)\left(\tilde{H}^{\dagger} \ell_{L}^{\kappa}\right)
$$


This operator ${ }^{1}$ violates global Lepton number, $\mathrm{U}(1)_{\mathrm{L}}$, which is accidentally preserved in the SMEFT operators of mass dimension less than or equal to four [11, 12]. For this reason it is generally neglected in LHC studies of the SMEFT. A nonzero value of this operator's Wilson coefficient leads to Majorana neutrino mass terms, which are not present in the minimal SM Lagrangian. Masses for neutrinos are now strongly experimentally supported [13] which makes it appealing to obtain a nonzero Wilson coefficient for this operator. Arguably the simplest way to generate this Wilson coefficient is to directly integrate out heavy singlet fields extending the SM (here denoted $N_{p}$ ) using a seesaw mechanism of neutrino mass generation [14-17]. A seesaw mechanism for neutrino mass provides an explanation of the smallness of neutrino masses due to a hierarchy of scales. Such an extension of the SM is well described by an effective field theory approach for the same reason.

In this paper, we systematically develop the SMEFT implementation and matching of the seesaw model, integrating out the heavy $N_{p}$ states assuming a renormalizable ultraviolet (UV) extension to the SM. We examine the effect of higher dimensional operators in the SMEFT operator expansion, beyond the Weinberg operator, on the low energy neutrino mass matrix that results. We find by explicit calculation the tree level matching contributions to the SMEFT dimension seven operators.

The seesaw model has been studied many times in the past in an EFT context, see refs. [18-28]. Our results go beyond past work by reporting the complete matching for three generations of heavy singlet fields integrated out in sequence in the seesaw model for the first time up to dimension seven. Simultaneously we incorporate into this implementation of neutrino EFT the flavour space expansion of neutrino phenomenology previously developed in ref. [29]. We discuss how flavour space expansions can be used to relate the neutrino mass spectrum to the Pontecorvo-Maki-Nakagawa-Sakata (PMNS) matrix [30, 31] phenomenology. We extend the results of ref. [29] using the fact that one can perturb neutrino phenomenology about the eigenvectors diagonalizing the lepton mass matrix in a general way, simply treating these vectors as an unknown basis of $\mathbb{C}^{3}$. We demonstrate the utility of the systematic expansion that can be constructed using this technique with a simple phenomenological example.

The method developed here can be used to study the growing data set on neutrino phenomenology. This can be done in a systematically improvable manner, using well defined expansions, in an effective field theory approach. This formalism is sufficiently general that it can accommodate flavour symmetries assumed in the UV sector, but is not limited to any such flavour symmetry requirement. This approach can also be extended to other UV models of neutrino mass generation in a straightforward manner.

\footnotetext{
${ }^{1}$ The $c$ superscript in eq. (1.1) corresponds to a charge conjugated Dirac four component spinor defined as $\psi^{c}=C \bar{\psi}^{T}$ with $C=-i \gamma_{2} \gamma_{0}$ in the chiral basis we employ. The star superscript is reserved for the complex conjugation operation that is applied to scalar and vector quantities. Chiral projection and charge conjugation do not commute (see the discussion in ref. [10] for a review). $\ell_{L}^{c}$ denotes the doublet lepton field that is chirally projected and subsequently charge conjugated.
} 


\section{Full theory for a minimal seesaw scenario}

We consider the full theory Lagrangian ${ }^{2}$ as given by $\mathcal{L}=\mathcal{L}_{\mathrm{SM}}+\mathcal{L}_{N_{p}}$. To fix our notation we define the SM Lagrangian $\left(\mathcal{L}_{\mathrm{SM}}\right)$ as

$$
\begin{aligned}
\mathcal{L}_{\mathrm{SM}}= & -\frac{1}{4}\left(G_{\mu \nu}^{A} G^{A \mu \nu}+W_{\mu \nu}^{I} W^{I \mu \nu}+B_{\mu \nu} B^{\mu \nu}\right)+\left(D_{\mu} H\right)^{\dagger}\left(D^{\mu} H\right)+\sum_{\psi} \bar{\psi} i \not D \psi \\
& -\left(H^{\dagger j} \bar{d}_{R} Y_{d} Q_{L j}+\tilde{H}^{\dagger j} \bar{u}_{R} Y_{u} Q_{L j}+H^{\dagger j} \bar{e}_{R} Y_{e} \ell_{L_{j}}+\text { h.c. }\right)-\lambda\left(H^{\dagger} H-\frac{1}{2} v^{2}\right)^{2} .
\end{aligned}
$$

Here the fermion fields summed over are $\psi=\left\{Q_{L}, u_{R}, d_{R}, \ell_{L}, e_{R}\right\}$ and the fields in $\mathcal{L}_{\mathrm{SM}}$ are written in the weak eigenstate basis. $\tilde{H}_{j}=\epsilon_{j k} H^{\dagger k}$, where $\epsilon_{12}=1$ and $\epsilon_{j k}=-\epsilon_{k j}, j, k=$ $\{1,2\} . H^{j}$ is the Higgs field of the $\mathrm{SM}$ with labeled $\mathrm{SU}(2)_{\mathrm{L}}$ components, conventionally indicated with Roman letters, usually $\{j, k, l, m, n\}$ in this work. At times we suppress the explicit $\mathrm{SU}(2)_{\mathrm{L}}$ indicies on the $\epsilon_{i j}$ tensor. The Higgs mass is defined as $m_{H}^{2}=2 \lambda v^{2}$. The fermion mass matrices are $M_{u, d, e}=Y_{u, d, e} v / \sqrt{2}$. The $M_{u, d, e}$ and Yukawa matrices $Y_{u, d, e}$ are complex matrices in flavour space. The gauge covariant derivative is defined as

$$
D_{\mu}=\partial_{\mu}+i g_{3} T^{A} A_{\mu}^{A}+i g_{2} t^{I} W_{\mu}^{I}+i g_{1} \mathrm{y} B_{\mu},
$$

where $T^{A}$ are the $\mathrm{SU}(3)$ generators, $t^{I}=\tau^{I} / 2$ are the $\mathrm{SU}(2)$ generators, and $\mathrm{y}$ is the $\mathrm{U}(1)$ hypercharge generator. Flavour indicies are suppressed in eq. (2.1), restoring the flavour indicies one has for example: $H^{\dagger j} \bar{d} Y_{d} q_{j} \rightarrow H^{\dagger j} \bar{d}_{p}\left[Y_{d}\right]_{p r} q_{r j}$ where the flavor indicies (conventionally $p, r, s, t)$ are summed over $\{1,2,3\}$ for the three generations.

The extension of the SM Lagrangian from a right handed singlet field $N_{R}$ with vanishing $\mathrm{SU}(3) \times \mathrm{SU}(2)_{\mathrm{L}} \times \mathrm{U}(1)_{\mathrm{Y}}$ charge is well known. Such fields can have Majorana mass terms [32] of the form

$$
\overline{N_{R, p}^{c}} M_{p r} N_{R, r}+\overline{N_{R, p}} M_{p r}^{\star} N_{R, r}^{c},
$$

where the charge conjugate of $N_{R}$ is $N_{R}^{c}$. Following ref. [18], we define a field satisfying the Majorana condition $N_{p}=N_{p}^{c}$ in its mass eigenstate basis, with all Majorana phases $\theta_{p}$ for each real mass eigenstate shifted into the effective couplings [18],

$$
N_{p}=e^{i \theta_{p} / 2} N_{R, p}+e^{-i \theta_{p} / 2}\left(N_{R, p}\right)^{c} .
$$

The corresponding Lagrangian is defined as

$$
2 \mathcal{L}_{N_{p}}=\overline{N_{p}}\left(i \not \partial-m_{p}\right) N_{p}-\overline{\ell_{L}^{\beta}} \tilde{H} \omega_{\beta}^{p, \dagger} N_{p}-\overline{\ell_{L}^{c \beta}} \tilde{H}^{*} \omega_{\beta}^{p, T} N_{p}-\overline{N_{p}} \omega_{\beta}^{p, *} \tilde{H}^{T} \ell_{L}^{c \beta}-\overline{N_{p}} \omega_{\beta}^{p} \tilde{H}^{\dagger} \ell_{L}^{\beta}
$$

The $\omega_{\beta}^{p}=\left\{x_{\beta}, y_{\beta}, z_{\beta}\right\}$ are each complex vectors in flavour space that have absorbed the Majorana phases. The invariants constructed from these vectors will allow a flavour space expansion as we discuss below. $N_{p}$ is a four component spinor satisfying the Majorana condition, not a two component Weyl spinor. We use greek letters such as $\beta, \kappa$ for the label of a flavour vector in the heavy singlet field mass eigenbasis.

\footnotetext{
${ }^{2}$ We acknowledge that explicit mass scales are introduced without a dynamical origin in this "full theory" - castigat ridendo mores.
} 


\subsection{Equations of motion of the seesaw theory}

We integrate out each $N_{p}$ in sequence, and utilize the Equation of Motion (EOM) to reduce to an operator basis. The EOM include the $N_{p}$ states still present in the spectrum. The relevant modifications of the SM EOM are

$$
\begin{aligned}
D^{2} H_{j}= & \lambda\left(v^{2}-2\left(H^{\dagger} H\right)\right) H_{j}-\bar{Q}_{L}^{k} Y_{u}^{\dagger} u_{R} \epsilon_{k j}-\bar{d}_{R} Y_{d} Q_{L j}-\bar{e}_{R} Y_{e} \ell_{L j}, \\
& -\frac{1}{2} \overline{\ell_{L}}{ }^{k \beta} \epsilon_{k j}\left(\omega_{\beta}^{p}\right)^{\dagger} N_{p}+\frac{1}{2} \overline{N_{p}} \omega_{\beta}^{p, *} \epsilon_{j k} \ell_{L}^{c, k \beta},
\end{aligned}
$$

and

$$
i \not D\left(\ell_{L}^{j}\right)_{\beta}=\left(Y_{e}^{\dagger}\right)_{\beta s} e_{R}^{s} H^{j}+\frac{1}{2} \tilde{H}^{j} \omega_{\beta}^{p, \dagger} N_{p}+\frac{c_{\beta \kappa}}{2} H^{j}\left(\tilde{H}^{T} \ell_{L}^{c, \kappa}\right)
$$

Note the last term in the EOM due to varying the fields in the $\mathcal{L}_{5}$ operator in the SMEFT. ${ }^{3}$ Finally the EOM for the $N_{p}$ are

$$
\not \partial N_{p}=-i\left(m_{p} N_{p}+w_{\beta}^{p, *} \tilde{H}^{T} \ell_{L}^{c \beta}+w_{\beta}^{p} \tilde{H}^{\dagger} \ell_{L}^{\beta}\right) .
$$

The usage of the EOM consistently drops a $N_{p}$ field when it is integrated out of the theory.

\section{Matching the seesaw to the SMEFT}

Integrating out the $N_{p}$ we match onto the SMEFT. The SMEFT is defined as the sum of $\mathrm{SU}(3)_{\mathrm{C}} \times \mathrm{SU}(2)_{\mathrm{L}} \times \mathrm{U}(1)_{\mathrm{Y}}$ invariant higher dimensional operators built out of SM fields

$$
\mathcal{L}_{\mathrm{SMEFT}}=\mathcal{L}_{\mathrm{SM}}+\mathcal{L}^{(5)}+\mathcal{L}^{(6)}+\mathcal{L}^{(7)}+\ldots, \quad \mathcal{L}^{(k)}=\sum_{\alpha=1}^{n_{k}} \frac{C_{\alpha}^{(k)}}{\Lambda^{k-4}} \mathcal{Q}_{\alpha}^{(k)} \quad \text { for } k>4 .
$$

Here $\mathcal{L}^{(k)}$ contains the dimension $k$ operators $\mathcal{Q}_{\alpha}^{(k)}$. The number of non redundant operators in $\mathcal{L}^{(5)}, \mathcal{L}^{(6)}, \mathcal{L}^{(7)}$ and $\mathcal{L}^{(8)}$ is known [1, 2, 4-9]. Past works on $\mathcal{L}^{(7)}$ operator bases particularly relevant to this study are refs. [1, 8, 9, 27, 33, 34].

Matching onto the SMEFT is defined by requiring that the Wilson coefficients in the higher dimensional operators reproduce the low energy, or infrared (IR), limit of the full theory. For example, consider the IR limit where $s^{2} \ll m_{p}^{2}$ for $N_{p}$ carrying four momenta $s^{\mu}$ as illustrated in figure 1 . The $N_{p} s$-channel propagator is expanded in this limit as

$$
\left(\$+m_{p}\right) \frac{-1}{m_{p}^{2}}\left(\frac{1}{1-s^{2} / m_{p}^{2}}\right)=-\frac{1}{m_{p}}-\frac{\$}{m_{p}^{2}}-\frac{s^{2}}{m_{p}^{3}}+\cdots
$$

Note that we adopt a conventional normalization of the Wilson coefficient of the dimension five operator of the form

$$
\mathcal{L}^{(5)}=\frac{c_{\beta \kappa}}{2} \mathcal{Q}_{5}^{\beta \kappa}+\text { h.c. }
$$

\footnotetext{
${ }^{3}$ Note that a series of $1 / m_{i}^{n}$ terms also exist correcting the right hand side of eq. (2.6) in the SMEFT, including correction due to $\mathcal{L}_{5}$, but these terms are supressed. The reason is that such corrections due to the Higgs EOM do not lead to $\mathcal{L}^{(7)}$ matching corrections. The dimensionality of the fields in the $\mathcal{L}^{(6)}$ matching only allow one derivative insertion, while the Higgs EOM has two derivatives.
} 


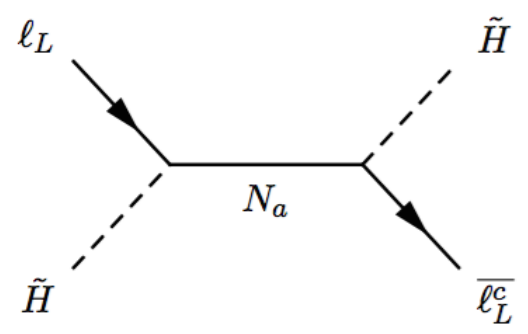

Figure 1. Tree level exchange expanded out to match onto $\mathcal{L}^{(5)}, \mathcal{L}^{(6)}, \mathcal{L}^{(7)} \ldots$.

\section{$3.1 \mathcal{L}^{(5)}$ matching}

Integrating out the heaviest $N_{p}$ state, denoted $N_{1}$, the matching onto the leading $\mathcal{L}^{(5)}$ operators is given by

$$
\mathcal{L}^{(5)}=\frac{\left(x_{\beta}\right)^{T} x_{\kappa}}{2 m_{1}} \mathcal{Q}_{5}^{\beta \kappa}+\text { h.c. }
$$

The matrix $\left(x_{\beta}\right)^{T} x_{\kappa} / m_{1}$ is complex with only one eigenvalue, as only $N_{1}$ was integrated out coupled to the complex flavour vector $x_{\beta}$. The notation $x_{\beta}^{T} x_{\kappa}$ is an outer product of the complex vectors. Integrating out the remaining two lighter $N_{p}$ states in sequence gives

$$
\mathcal{L}^{(5)}=\frac{c_{\beta \kappa}}{2} \mathcal{Q}_{5}^{\beta \kappa}+\text { h.c. }
$$

where $c_{\beta \kappa}=\left(\omega_{\beta}^{p}\right)^{T} \omega_{\kappa}^{p} / m_{p}$ and the flavour index $p$ is summed over. Contracting the $\mathrm{SU}(2)_{\mathrm{L}}$ indicies of $\mathcal{Q}_{5}$ and taking a matrix element where the Higgs field is taken as the background field value gives

$$
\left\langle c_{\beta \kappa} \mathcal{Q}_{5}^{\beta \kappa}\right\rangle=\frac{v^{2} c_{\beta \kappa}}{2} \overline{\nu_{L}^{c \beta}} \nu_{L}^{\kappa} .
$$

We define the mass eigenstate neutrino fields with prime superscripts. These field are related by the unitary rotation matricies (denoted $\mathcal{U}$ ) to the weak eigenstates used so far by

$$
\nu_{L}^{p}=\mathcal{U}(\nu, L)_{r}^{p} \nu_{L}^{\prime r},
$$

Changing to the mass eigenstate basis we find

$$
\left\langle c_{\beta \kappa} \mathcal{Q}_{5}^{\beta \kappa}\right\rangle=-\frac{v^{2}}{2}\left[\mathcal{U}^{T}(\nu, L)_{p}^{\beta} c_{\beta \kappa} \mathcal{U}(\nu, L)_{r}^{\kappa}\right]\left(\nu_{L}^{\prime}\right)^{T p} \epsilon\left(\nu_{L}^{\prime}\right)^{r}
$$

where $\mathcal{U}^{T}(\nu, L)_{p}^{\beta} c_{\beta \kappa} \mathcal{U}(\nu, L)_{r}^{\kappa} \equiv-\operatorname{diag}\left\{C^{1}, C^{2}, C^{3}\right\}_{p r}$. The physical low energy neutrino masses $m_{\nu}^{p}$ at leading order in the SMEFT expansion in $v / m_{p}$ are then given as ${ }^{4}$

$$
m_{\nu}^{p}=\frac{v^{2}}{2} C^{p}
$$

\footnotetext{
${ }^{4}$ The overall sign in the Majorana mass term is linked to the phase convention choice on $C$.
} 


\section{$3.2 \quad \mathcal{L}^{(6)}$ matching}

The $\mathcal{L}^{(6)}$ matching follows directly and we find

$$
\mathcal{L}^{(6)}=\frac{\left(\omega_{\beta}^{p}\right)^{\dagger} \omega_{\kappa}^{p}}{2 m_{p}^{2}}\left(\underset{\beta \kappa}{\mathcal{Q}_{H \ell}^{(1)}-\mathcal{Q}_{\beta \ell}^{(3)}}\right) .
$$

The operators $Q_{H \ell}^{(1)}, Q_{H \ell}^{(3)}$ each with flavour indicies $\beta \kappa$ are defined as in ref. [5] with the notation $\phi$ exchanged for $H$ for the Higgs field. ${ }^{5}$ Here we have reduced the operators to the Warsaw basis form using the EOM and combining terms into Hermitian derivatives defined as $H^{\dagger} i \overleftrightarrow{D}_{\beta} H=i H^{\dagger}\left(D_{\beta} H\right)-i\left(D_{\beta} H\right)^{\dagger} H$ and $H^{\dagger} i \overleftrightarrow{D}_{\beta}^{I} H=i H^{\dagger} \tau^{I}\left(D_{\beta} H\right)-i\left(D_{\beta} H\right)^{\dagger} \tau^{I} H$ We have used the fact that Hermitian operators generate real eigenvalues, and hence the matching coefficient in eq. (3.10) is real. The derivative on the lepton doublet field has been reduced out using the EOM and using the fact that $\tilde{H}^{\dagger} H=0$. For previous results on dimension six matching comparable to the terms in eq. (3.10) see refs. [18, 21, 22, 35]. Our results are distinct from past works in the $\mathrm{SU}(2)_{\mathrm{L}}$ field dependence. As eq. (2.6) contains the $N_{p}$ fields still in the spectrum when integrating out the heavy Majorana mass eigenstates in sequence, the following terms are also generated. Integrating out $N_{1}$ gives

$$
\begin{aligned}
\mathcal{L}_{N_{2,3}}^{(6), N_{1}} \supseteq & \frac{\operatorname{Re}\left[x_{\beta}^{\dagger} x^{\star} \cdot y^{\dagger}\right]}{4 m_{1}^{2}}\left(\mathcal{Q}_{N_{2}}^{\beta}-\mathcal{Q}_{N_{2}}^{\star, \beta}\right)+\frac{i \operatorname{Im}\left[x_{\beta}^{\dagger} x^{\star} \cdot y^{\dagger}\right]}{4 m_{1}^{2}}\left(\mathcal{Q}_{N_{2}}^{\beta}+\mathcal{Q}_{N_{2}}^{\star, \beta}\right), \\
& +\frac{\operatorname{Re}\left[x_{\beta}^{\dagger} x^{\star} \cdot z^{\dagger}\right]}{4 m_{1}^{2}}\left(\mathcal{Q}_{N_{3}}^{\beta}-\mathcal{Q}_{N_{3}}^{\star, \beta}\right)+\frac{i \operatorname{Im}\left[x_{\beta}^{\dagger} x^{\star} \cdot z^{\dagger}\right]}{4 m_{1}^{2}}\left(\mathcal{Q}_{N_{3}}^{\beta}+\mathcal{Q}_{N_{3}}^{\star, \beta}\right),
\end{aligned}
$$

integrating out $N_{2}$ gives

$$
\mathcal{L}_{N_{3}}^{(6), N_{2}} \supseteq \frac{\operatorname{Re}\left[y_{\beta}^{\dagger} y^{\star} \cdot z^{\dagger}\right]}{4 m_{2}^{2}}\left(\mathcal{Q}_{N_{3}}^{\beta}-\mathcal{Q}_{N_{3}}^{\star, \beta}\right)+\frac{i \operatorname{Im}\left[y_{\beta}^{\dagger} y^{\star} \cdot z^{\dagger}\right]}{4 m_{2}^{2}}\left(\mathcal{Q}_{N_{3}}^{\beta}+\mathcal{Q}_{N_{3}}^{\star, \beta}\right),
$$

where $\mathcal{Q}_{N_{p}}^{\beta}=\left(H^{\dagger} H\right)\left(\overline{\ell_{L}^{\beta}} \tilde{H}\right) N_{p}$. Here the notation $a \cdot b$ applied to complex flavour vectors $\{x, y, z\}$ is a Hermitian inner product, see the appendix for details on the flavour space algebra.

Due to the presence of the Majorana mass scale in the EOM the following contributions to $\mathcal{L}_{N_{2,3}}^{(6)}$ are also present. Integrating out $N_{1}$

$$
\mathcal{L}_{N_{2,3}}^{(6), N_{1}} \supseteq \frac{\left(x_{\beta}\right)^{T} x^{\star} \cdot y^{\dagger} m_{2}}{4 m_{1}^{3}}\left[\overline{\ell_{L \beta}^{c}} \tilde{H}^{\star} N_{2}\right]\left(H^{\dagger} H\right)+\frac{\left(x_{\beta}\right)^{T} x^{\star} \cdot z^{\dagger} m_{3}}{4 m_{1}^{3}}\left[\overline{\ell_{L \beta}^{c}} \tilde{H}^{\star} N_{3}\right]\left(H^{\dagger} H\right),
$$

while integrating out $N_{2}$ gives

$$
\mathcal{L}_{N_{3}}^{(6), N_{2}} \supseteq \frac{\left(y_{\beta}\right)^{T} y^{\star} \cdot z^{\dagger} m_{3}}{4 m_{2}^{3}}\left[\overline{\ell_{L \beta}^{c}} \tilde{H}^{\star} N_{3}\right]\left(H^{\dagger} H\right)+\text { h.c. }
$$

\footnotetext{
${ }^{5}$ Explicitly these operators are given by $\underset{\beta \kappa}{\mathcal{Q}_{H \ell}^{(1)}}=H^{\dagger} i \overleftrightarrow{D}{ }_{\mu} H \ell_{\beta} \gamma^{\mu} \ell_{\kappa}$ and $\underset{\beta \kappa}{\mathcal{Q}_{H \ell}^{(3)}}=H^{\dagger} i \overleftrightarrow{D_{\mu}^{I}} H \ell_{\beta} \gamma^{\mu} \tau_{I} \ell_{\kappa}$
} 


\section{$3.3 \quad \mathcal{L}^{(7)}$ matching}

Dimension seven operators come about due to the expansion of a propagator, such as eq. (3.2), to third order, and from the contraction of the local contact operators present in $\mathcal{L}_{N_{2,3}}^{(6)}$ once $N_{1,2}$ are integrated out in time ordered products. We follow the approach in refs. $[1,5]$ of removing derivative operators in the basis. We define the short hand notation to aid in presenting the results

$$
\tilde{C}_{\beta \kappa}^{7}=\sum_{p} \frac{\left(\omega_{\beta}^{p}\right)^{T} \omega_{\kappa}^{p}}{2 m_{p}^{3}} .
$$

Using the Higgs EOM in eq. (2.6) on the results of the tree level exchange of $N_{p}$ expanded to third order, one finds the terms

$$
\mathcal{L}^{(7)} \supseteq-\frac{\lambda v^{2} \tilde{C}_{\beta \kappa}^{7}}{2}\left(\overline{\ell_{L \beta}^{c}} \ell_{L \kappa}\right) H^{2}+2 \lambda \tilde{C}_{\beta \kappa}^{7} \mathcal{Q}_{\ell H}+\frac{\lambda v^{2} \tilde{C}_{\beta \kappa}^{7}}{2}\left(\overline{\ell_{L \beta}^{c}} \sigma^{I} \ell_{L \kappa}\right) H \sigma^{I} H+h . c
$$

$\mathcal{Q}_{L H}$ and the remaining operator notation for $\mathcal{L}^{(7)}$ is defined in ref. [1]. ${ }^{6}$ Eq. (3.16) vanishes when the Higgs takes on its background expectation value. This leads to a vanishing of the contributions to the low energy neutrino mass matrix from this sum of terms. Applying the Higgs EOM and reducing the direct matching contributions into field strengths of the SM fields leads to the $\mathcal{L}^{(7)}$ operators ${ }^{7}$

$$
\begin{aligned}
\mathcal{L}^{(7)} \supseteq & -\tilde{C}_{\beta \kappa}^{7} Y_{u}^{\dagger} \mathcal{Q}_{\ell \ell \bar{Q} u H}^{\kappa \beta}-\left(\tilde{C}_{\kappa \beta}^{7}-\tilde{C}_{\beta \kappa}^{7}\right) Y_{d} \mathcal{Q}_{\ell \ell Q \bar{d} H}^{(1) \beta \kappa}-\tilde{C}_{\beta \kappa}^{7} Y_{d} \mathcal{Q}_{\ell \ell Q \bar{d} H}^{(2) \beta \kappa}+\tilde{C}_{\beta \kappa}^{7} Y_{e} \mathcal{Q}_{\ell \ell \ell \bar{e} H}^{\kappa \beta}, \\
& +g_{1} y_{\ell} \tilde{C}_{\beta \kappa}^{7} \mathcal{Q}_{\ell H B}^{\beta \kappa}+\frac{g_{2} \tilde{C}_{\beta \kappa}^{7}}{2} \mathcal{Q}_{\ell H W}^{\beta \kappa}-i \tilde{C}_{\beta \kappa}^{7}\left(Y_{e}^{\dagger}\right)_{\kappa}^{\alpha} \mathcal{Q}_{\ell H D e_{\alpha}}^{\beta}+\frac{\left(x_{\beta}\right)^{T} x^{\star} \cdot y^{\dagger} y_{\delta}}{4 m_{1}^{3}} \mathcal{Q}_{\ell H}^{\beta \delta}, \\
& +\frac{\left(x_{\beta}\right)^{T} x^{\star} \cdot z^{\dagger} z_{\delta}}{4 m_{1}^{3}} \mathcal{Q}_{\ell H}^{\beta \delta}+\frac{\left(y_{\beta}\right)^{T} y^{\star} \cdot z^{\dagger} z_{\delta}}{4 m_{2}^{3}} \mathcal{Q}_{\ell H}^{\beta \delta}-2 \tilde{C}_{\beta \kappa}^{7} \mathcal{Q}_{\ell H D}^{(2)}+h . c .
\end{aligned}
$$

Here we have used Fierz relations and the EOM to reduce to this basis, utilizing refs. [33, 37, 38]. It is also important to include the effect of $\mathcal{L}_{5}$ in determining the EOM for the lepton fields, as this contribution leads to a matching contribution to $\mathcal{Q}_{\ell H}$ of the form

$$
\begin{aligned}
\mathcal{L}^{(7)} \supseteq & -\left[\frac{x_{\beta}^{T} x_{\kappa}\|x\|}{4 m_{1}^{3}}+\frac{y_{\beta}^{T} y_{\kappa}\|y\|}{4 m_{2}^{3}}+\frac{z_{\beta}^{T} z_{\kappa}\|z\|}{4 m_{3}^{3}}\right] \mathcal{Q}_{\ell H}, \\
& -\left[\frac{x_{\beta}^{T} y_{\kappa} y \cdot x}{4 m_{2}^{2} m_{1}}+\frac{x_{\beta}^{T} z_{\kappa} z \cdot x}{4 m_{3}^{2} m_{1}}+\frac{y_{\beta}^{T} z_{\kappa} z \cdot y}{4 m_{3}^{2} m_{2}}\right] \mathcal{Q}_{\ell H}+\text { h.c. }
\end{aligned}
$$

\footnotetext{
${ }^{6}$ The explicit operator definitions for $\mathcal{L}^{(7)}$ are listed in the appendix for completeness.

${ }^{7}$ Note that the renormalizable weakly coupled seesaw model induces operators with field strengths in $\mathcal{L}^{(7)}$ at tree level. This is expected on general grounds in well defined EFT's [36].
} 
This contribution perturbs the neturino mass matrix, as we discuss below. The operators in $\mathcal{L}_{N_{2,3}}^{(7)}$ when $N_{1}$ is integrated out are given by

$$
\begin{aligned}
\mathcal{L}_{N_{2,3}}^{(7)} \supseteq & \frac{\left(x_{\beta}\right)^{T} x_{\kappa} y_{\alpha}^{\dagger}}{8 m_{1}^{3}}\left[\overline{\ell_{L \beta}^{c}} \ell_{L \kappa}\right] \overline{\ell_{L}^{\alpha}} \tilde{H}^{\star} N_{2}+\frac{\left(x_{\beta}\right)^{T} x_{\kappa} z_{\alpha}^{\dagger}}{8 m_{1}^{3}}\left[\overline{\ell_{L \beta}^{c}} \ell_{L \kappa}\right] \overline{\ell_{L}^{\alpha}} \tilde{H}^{\star} N_{3}, \\
& +\frac{\left(x_{\beta}\right)^{T} x_{\kappa} y_{\alpha}^{\dagger}}{8 m_{1}^{3}}\left[\overline{\ell_{L \beta}^{c}} \sigma^{I} \ell_{L \kappa}\right] \overline{\ell_{L}^{\alpha}} \sigma^{I} \tilde{H}^{\star} N_{2}+\frac{\left(x_{\beta}\right)^{T} x_{\kappa} z_{\alpha}^{\dagger}}{8 m_{1}^{3}}\left[\overline{\ell_{L \beta}^{c}} \sigma^{I} \ell_{L \kappa}\right] \overline{\ell_{L}^{\alpha}} \sigma^{I} \tilde{H}^{\star} N_{3}, \\
& +\frac{\left(x_{\beta}\right)^{T} x_{\kappa} y_{\alpha}^{\star}}{8 m_{1}^{3}}\left[\overline{\ell_{L \beta}^{c}} \ell_{L \kappa}\right] \overline{N_{2}} \ell_{L \alpha}^{c} \tilde{H}^{\star}+\frac{\left(x_{\beta}\right)^{T} x_{\kappa} z_{\alpha}^{\star}}{8 m_{1}^{3}}\left[\overline{\ell_{L \beta}^{c}} \ell_{L \kappa}\right] \overline{N_{3}} \ell_{L \alpha}^{c} \tilde{H}^{\star}, \\
& +\frac{\left(x_{\beta}\right)^{T} x_{\kappa} y_{\alpha}^{\star}}{8 m_{1}^{3}}\left[\overline{\ell_{L \beta}^{c}} \sigma^{I} \ell_{L \kappa}\right] \overline{N_{2}} \sigma^{I} \ell_{L \alpha}^{c} \tilde{H}^{\star}+\frac{\left(x_{\beta}\right)^{T} x_{\kappa} z_{\alpha}^{\star}}{8 m_{1}^{3}}\left[\overline{\ell_{L \beta}^{c}} \sigma^{I} \ell_{L \kappa}\right] \overline{N_{3}} \sigma^{I} \ell_{L \alpha}^{c} \tilde{H}^{\star}, \\
& +\frac{i\left(x_{\beta}\right)^{T} x^{\star} \cdot y^{\dagger}}{4 m_{1}^{3}}\left[\overline{\ell_{L \beta}^{c}} \gamma_{\mu} N_{2}\right] \tilde{H}^{\star}\left(H D_{\mu} H^{\dagger}\right)+\frac{i\left(x_{\beta}\right)^{T} x^{\star} \cdot z^{\dagger}}{4 m_{1}^{3}}\left[\overline{\ell_{L \beta}^{c}} \gamma_{\mu} N_{3}\right] \tilde{H}^{\star}\left(H D_{\mu} H^{\dagger}\right)+\text { h.c. }
\end{aligned}
$$

In addition, when $N_{2}$ is integrated out in sequence the additional matching contributions to the operators involving $N_{3}$ are

$$
\begin{aligned}
\mathcal{L}_{N_{3}}^{(7)} \supseteq & \frac{\left(y_{\beta}\right)^{T} y_{\kappa} z_{\alpha}^{\dagger}}{8 m_{2}^{3}}\left[\overline{\ell_{L \beta}^{c}} \ell_{L \kappa}\right] \overline{\ell_{L}^{\alpha}} \tilde{H}^{\star} N_{3}+\frac{\left(y_{\beta}\right)^{T} y_{\kappa} z^{\star}}{8 m_{2}^{3}}\left[\overline{\ell_{L \beta}^{c}} \ell_{L \kappa}\right] \overline{N_{3}} \ell_{L \alpha}^{c} \tilde{H}^{\star}, \\
& +\frac{\left(y_{\beta}\right)^{T} y_{\kappa} z_{\alpha}^{\dagger}}{8 m_{2}^{3}}\left[\overline{\ell_{L \beta}^{c}} \sigma^{I} \ell_{L \kappa}\right] \overline{\ell_{L}^{\alpha}} \sigma^{I} \tilde{H}^{\star} N_{3}+\frac{\left(y_{\beta}\right)^{T} y_{\kappa}}{8 m_{2}^{3}}\left[\overline{\ell_{L \beta}^{c}} \sigma^{I} \ell_{L \kappa}\right] \overline{N_{3}} \sigma^{I} \ell_{L \alpha}^{c} \tilde{H}^{\star}, \\
& +\frac{i\left(y_{\beta}\right)^{T} y^{\star} \cdot z^{\dagger}}{4 m_{2}^{3}}\left[\overline{\ell_{L \beta}^{c}} \gamma_{\mu} N_{3}\right] \tilde{H}^{\star}\left(H D_{\mu} H^{\dagger}\right)+\text { h.c. }
\end{aligned}
$$

We have checked the $\mathcal{L}^{(5,6,7)}$ matching results with multiple matrix elements to avoid any potential matching ambiguities. We also note the $N_{p}$ mass matrix gets perturbed after integrating out $N_{1}$ or $N_{2}$. We have determined these corrections, but as they are dimension eight in the SMEFT they are neglected here.

\section{Perturbation and non-perturbation of the neutrino mass matrix}

At tree level if the $N_{p}$ states are integrated out simultaneously or not, the low energy neutrino mass matrix is perturbed due to $\mathcal{L}^{(7)}$ matchings. The nature of the perturbations are however reflective of the orientations of the heavy singlet fields in flavour space, as well as their mass spectrum.

It is interesting that a number of effects that would perturb the low energy neutrino mass matrix cancel out. For example, the terms in eq. (3.16) cancel in the limit that the Higgs takes on its vacuum expectation value, as previously mentioned. Integration by parts and EOM manipulations can be used to see this result in the complete basis, when considering the matching onto the operator $\mathcal{Q}_{\ell H}$. This operator does lead to a contribution 


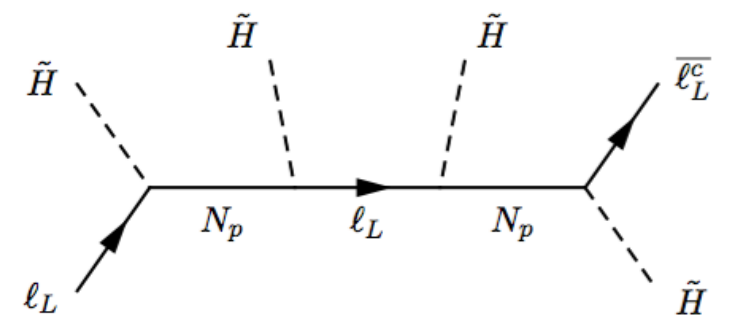

Figure 2. Full theory interactions inserted on the tree level propagator to obtain four external $H$ fields. These scattering contributions only perturb the low energy neutrino mass matrix due to the EOM effect of $\mathcal{L}_{5}$ modifying eq. (2.7). Note that when the neutrinos are integrated out in sequence, local contact operators result that lead to even more mass matrix perturbations, as shown in figure 3 .

to the neutrino mass matrix when the Higgs takes on its vacuum expectation value

$$
\left\langle C_{\ell H}^{\beta \kappa} \epsilon_{i j} \epsilon_{m n}\left(\ell_{L}^{i \beta} C \ell_{L}^{m \kappa}\right) H^{j} H^{n}\left(H^{\dagger} H\right)\right\rangle=-\frac{v^{4} C_{\ell H}^{\beta \kappa}}{4}\left(\nu_{L}^{\beta}\right)^{T} \epsilon \nu_{L}^{\kappa} .
$$

The dependence on this operator in the expansion of the propagator to third order can be seen to vanish integrated by parts, while also using eq. (2.8). One finds

$$
\frac{i\left(x_{\beta}\right)^{T} x^{\star} \cdot y^{\dagger}}{4 m_{1}^{3}}\left[\overline{\ell_{L \beta}^{c}} \gamma_{\mu} N_{2}\right] \tilde{H}^{\star}\left(H D_{\mu} H^{\dagger}\right) \rightarrow-\frac{\left(x_{\beta}\right)^{T} x^{\star} \cdot y^{\dagger} y_{\delta}}{4 m_{1}^{3}} \mathcal{Q}_{\ell H}^{\beta \delta}+\cdots
$$

which cancels the corresponding $\mathcal{Q}_{\ell H}$ term in eq. (3.17). No additional terms that contribute to the neutrino mass matrix result from the manipulations in the previous equation; these manipulations also cancel the terms in eq. (3.13). Alternatively, one can integrate out $N_{2,3}$ using the interactions in eq. (3.13). Doing so, one finds a contribution to $\mathcal{L}^{(7)}$ that directly cancels the $\mathcal{Q}_{\ell H}$ dependence in eq. (3.17). It is important to include $\mathcal{L}_{N_{2}, N_{3}}^{(6)}$ and $\mathcal{L}_{N_{2}, N_{3}}^{(7)}$ when defining the matching onto the theory to sub-leading order for this reason. Use of the EOM, and integration by parts on the $N_{p}$ states still in the spectrum when $N_{1}$ is integrated out leads to ambiguities if the full Lagrangian is not specified.

The fact that a subset of contributions to $\mathcal{L}^{(7)}$ related to the expansion of the propagator does not lead to a perturbation of the neutrino mass matrix at tree level can also been understood intuitively. To obtain $H^{\dagger} H$ times $\mathcal{Q}_{5}$ requires two extra insertions of the coupling of the $N_{p}$ states to the SM fields. In eq. (2.5) this coupling is always accompanied by the light SM field $\ell$ so that no local operator is obtained in the heavy $N_{p}$ limit expanding the propagator in Feynman diagrams, as illustrated in figure 2. This viual argument is limited, as this fact is not preserved when reducing the operators obtained in the expansion of the propagator by the EOM. This is another example of the fact that EOM effects in a field theory do not have a trivial Feynman diagram interpretation.

The detailed nature of the neutrino mass matrix perturbations do change if the states are integrated out simultaneously or not, as we discuss below. 


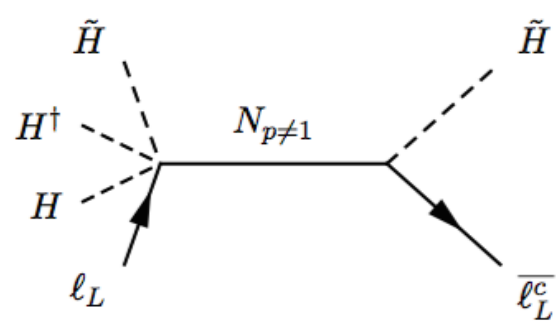

Figure 3. Tree level exchange corresponding to the time ordered product $\mathcal{T}\left(\mathcal{L}^{6}(x), \mathcal{L}^{4}(y)\right)$ generating a perturbation to the neutrino mass matrix.

\subsection{Time ordered products of $\mathcal{L}^{(6)}$ and $\mathcal{L}^{(4)}$}

The limited argument in the previous section also does not forbid perturbations of the neutrino mass matrix due to integrating out the $N_{p}$ states in sequence. Directly expanding out the propagator at tree level to third order, a $\mathcal{L}^{(7)}$ matching contribution comes about due to integrating out the heaviest $N_{p}$ mass eigenstate, and subsequently integrating out the lighter $N_{p}$ mass eigenstates. This always occurs as the $N_{p}$ cannot be indistinguishable and generate three distinct eigenvalues of the low energy neutrino mass matrix in the UV scenario we consider. (Different masses of the $N_{p}$ states alone still lead to only one low energy eigenvalue of the neutrino mass matrix, only with a different normalization.) These contribution also match onto $\mathcal{L}^{(7)}$ and lead to additional effects perturbing the neutrino mass matrix. The action in the EFT generated when the heaviest $N_{p}$ state is integrated out has a time ordered product contribution of the form

$$
S_{\text {eff }}=-\frac{1}{2} \int d x^{4} \int d y^{4} \mathcal{T}\left(\mathcal{L}_{N_{2,3}}^{6}(x), \mathcal{L}^{4}(y)\right)
$$

Reproducing the IR limit in the SMEFT give the following matching contributions

$$
\begin{aligned}
\mathcal{L}^{(7)} \supseteq & -\left(\frac{x_{\beta}^{T} y_{\alpha} x \cdot y}{4 m_{1}^{2} m_{2}}+\frac{x_{\beta}^{T} z_{\alpha} x \cdot z}{4 m_{1}^{2} m_{3}}+\frac{y_{\beta}^{T} z_{\alpha} y \cdot z}{4 m_{2}^{2} m_{3}}\right) \mathcal{Q}_{\ell H}^{\beta \alpha}, \\
& -\left(\frac{x_{\beta}^{T} y_{\alpha} y \cdot x}{4 m_{2}^{2} m_{1}}+\frac{x_{\beta}^{T} z_{\alpha} z \cdot x}{4 m_{3}^{2} m_{1}}+\frac{y_{\beta}^{T} z_{\alpha} z \cdot y}{4 m_{3}^{2} m_{2}}\right) \mathcal{Q}_{\ell H}^{\beta \alpha}+\text { h.c. }
\end{aligned}
$$

Note that the terms in the second line are generated when consistently retaining EOM terms (including the $N_{p}$ that remain in the spectrum) to reduce the matching contributions to a minimal basis. Again, for the heavy states to be indistinguishible and integrated out simultaneously, they would have to be oriented in flavour space in the same manner, and have identical masses. Note that these perturbations to the neutrino mass matrix are proportional to these differences in flavour space and the multiple mass scales. 


\subsection{The neutrino mass matrix up to $\mathcal{L}^{(7)}$}

The contributions to the neutrino mass matrix in the weak eigenbasis up to $\mathcal{L}^{(7)}$ are given $b^{8}$

$$
\begin{aligned}
\mathcal{L}_{\nu \nu}= & \frac{M_{\nu \nu}^{\beta \kappa}}{2} \nu_{L}^{T, \beta} \epsilon \nu_{L}^{\kappa}, \\
= & -\left[\frac{x_{\beta}^{T} x_{\kappa}}{m_{1}}\left(1+\frac{v^{2}\|x\|^{2}}{4 m_{1}^{2}}\right)+\frac{y_{\beta}^{T} y_{\kappa}}{m_{2}}\left(1+\frac{v^{2}\|y\|^{2}}{4 m_{2}^{2}}\right)+\frac{z_{\beta}^{T} z_{\kappa}}{m_{3}}\left(1+\frac{v^{2}\|z\|^{2}}{4 m_{3}^{2}}\right)\right] \frac{v^{2}}{4} \nu_{L}^{T, \beta} \epsilon \nu_{L}^{\kappa}, \\
& -\left[\frac{x_{\beta}^{T} y_{\kappa}}{m_{1} m_{2}}\left(\frac{x \cdot y}{m_{1}}+\frac{y \cdot x}{m_{2}}\right)+\frac{y_{\beta}^{T} z_{\kappa}}{m_{2} m_{3}}\left(\frac{y \cdot z}{m_{2}}+\frac{z \cdot y}{m_{3}}\right)\right] \frac{v^{4}}{16} \nu_{L}^{T, \beta} \epsilon \nu_{L}^{\kappa}, \\
& -\left[\frac{x_{\beta}^{T} z_{\kappa}}{m_{1} m_{3}}\left(\frac{x \cdot z}{m_{1}}+\frac{z \cdot x}{m_{3}}\right)\right] \frac{v^{4}}{16} \nu_{L}^{T, \beta} \epsilon \nu_{L}^{\kappa}+\text { h.c. }
\end{aligned}
$$

As the mass matrix is perturbed due to corrections at $\mathcal{L}^{(7)}$ which are suppressed by $\mathcal{O}\left(v^{4} / m_{p}^{3}\right)$ and of order $\mathcal{O}\left(\omega^{4}\right)$. As such it is established that these corrections can be neglected until perturbations of the Wilson coefficient in $\mathcal{L}^{(5)}$ is pushed to relative order $\mathcal{O}\left(\omega^{2} v^{2} / m_{p}^{2}\right)$ compared to leading effects captured by $\mathcal{Q}_{5}$. Radiative corrections to $\mathcal{Q}_{5}$ are generally larger than the non-pertubative corrections due to $\mathcal{L}_{7}$ and must be incorporated for phenomenological studies as well if these corrections are to be considered.

\section{$5 \quad$ Flavour space expansion for the eeesaw}

The expansion that results when integrating out the heavy singlet states in sequence is not the only expansion present in lower energy Neutrino phenomenology. The usual matching that was developed in the previous sections leads to small perturbations on the neutrino mass matrix. A larger effect for phenomenology is expanding the Wilson coefficient of the Weinberg operator systematically due to the perturbations of integrating out the $N_{1,2,3}$ states. In the remainder of this work, we incorporate and improve on results of ref. [29] to develop perturbations of the $\mathcal{U}(\nu, L)$ matricies, assuming a seesaw origin of neutrino mass. We use the SMEFT treatment of the seesaw model developed in the previous sections. The idea is to link perturbations of the PMNS matrix to perturbations of the neutrino mass generation mechanism. ${ }^{9}$ A key point underlying this approach is Majorana mass terms, unlike Dirac mass terms, originate in bi-linears of the same field operators. As such, the complex mass matrix is diagonalized by a single rotation matrix of the field $\nu_{L}$ introduced through $\nu_{L}^{p}=\mathcal{U}(\nu, L)^{p r} \nu_{L, r}^{\prime}$. For this reason any expansion of the neutrino mass matrix is more directly tied to an expansion of the unitary rotation matricies $\mathcal{U}(\nu, L)$.

With the results of the previous section, the Flavour Space Expansion (FSE) of ref. [29] is now on a firmer theoretical footing. For example, the heaviest neutrino in the low energy theory is generically linked to integrating out the lightest singlet field (denoted in this work $N_{3}$ ). In ref. [29] the neutrino mass matrix is generated by first integrating out the lightest

\footnotetext{
${ }^{8}$ Note that the overall sign in these terms is due to a convention choice on $C$.

${ }^{9}$ See also the related (but distinct) sequential dominance idea of S. King discussed in refs. [39-42].
} 
singlet state, and then integrating out the heavier $N_{p}$ in sequence. Although this can be done, it is conceptually more clear to integrate out the three $N_{p}$ states by removing the heaviest state first, as done here, and subsequently perturb the low energy neutrino mass matrix after the lighter $N_{p}$ states are removed in sequence. Doing so the usual SMEFT expansion is present, clarifying the impact of the multiple expansions present on low energy neutrino phenomenology.

\subsection{Developing the FSE}

The FSE is distinct from the double expansion in $\left(v / m_{p}\right)^{n}$, and $\left(E / m_{p}\right)^{n}$ that dictates the relative size of contributions in the SMEFT operator expansion. This eigenvector perturbation formalism can always be implemented in a type one seesaw model. However, there is no guarantee that the FSE will be quickly convergent, and therefore predictive, as it depends upon unknown UV physics parameters.

The basic expectation is that in seesaw models the FSE will be perturbative [29]. The reason is that the matrix $M_{p r}$ is expected to be approximately uniform in entries in the $N_{p}$ interaction eigenbasis to the SM states, as the $N_{p}$ do not carry (known SM) quantum numbers. Diagonalizing the corresponding mass matrix, any hierarchy in the couplings of the $N_{R}$ states to the SM states is washed out rotating the the mass eigenbasis, and the magnitude of the $\omega_{p}$ are drawn together. For this reason it is expected that

$$
\|x\| \sim\|y\| \sim\|z\|
$$

on general grounds. Here the notation refers to the Euclidean norm of the complex vectors in flavour space. The FSE is of the form

$$
M_{\nu \nu}^{\beta \alpha}\left(M_{\nu \nu}^{\kappa \alpha}\right)^{\dagger} \simeq \frac{\left\|z^{\star} \cdot z\right\|}{m_{3}^{2}}\left[z_{\beta}^{T} z_{\kappa}+\frac{z^{\star} \cdot y^{\dagger}}{\left\|z^{\star} \cdot z\right\|} \frac{m_{3}}{m_{2}} z_{\beta}^{T} y_{\kappa}^{\star}+\frac{y^{\star} \cdot z^{\dagger}}{\left\|z^{\star} \cdot z\right\|} \frac{m_{3}}{m_{2}} y_{\beta}^{T} z_{\kappa}^{\star}+\cdots\right] .
$$

The utility of the FSE depends upon

$$
\frac{z^{\star} \cdot y^{\dagger}}{\left\|z^{\star} \cdot z\right\|} \frac{m_{3}}{m_{2}}<1, \quad \frac{y^{\star} \cdot z^{\dagger}}{\left\|z^{\star} \cdot z\right\|} \frac{m_{3}}{m_{2}}<1,
$$

with similar conditions for integrating out the state of mass $m_{1}$. By construction the SMEFT matching has been formulated so that $\frac{m_{3}}{m_{2}}<1$. Using the Cauchy-Schwarz equality $a \cdot b=\Delta_{a b}\|a\|\|b\|$ with $\Delta_{a b}<1$ the FSE depends upon

$$
\frac{\|y\|}{\|z\|} \Delta_{y^{\dagger} z}<m_{2} / m_{3}, \quad \frac{\|y\|}{\|z\|} \Delta_{y z^{\dagger}}<m_{2} / m_{3} .
$$

Considering eq. (5.1), which directly follows from the quantum numbers of the $N_{p}$ states when tuning is avoided, it is expected that the FSE is present and convergent. In what follows we assume this is the case. Assuming the FSE exists, the results of ref. [29] follow directly, and can be expanded upon in the following way. To establish notation we define

$$
M_{\nu \nu}=U(\nu, L)^{\star} \operatorname{diag}\left(m_{c}, m_{b}, m_{a}\right) U(\nu, L)^{\dagger} .
$$


The rotation matrix is decomposed in eigenvectors such that $U(\nu, L)=\left(\vec{\rho}_{c}^{\star}, \vec{\rho}_{b}^{\star}, \vec{\rho}_{a}^{\star}\right)$ with $\vec{\rho}_{i}^{\star}$ a column vector with $\left\|\vec{\rho}_{i}\right\|=1$, and $\left\|\vec{\rho}_{i}^{\star}\right\|=1$. These eigenvectors are such that

$$
M_{\nu \nu} \vec{\rho}_{p}^{\star}=m_{p} \vec{\rho}_{p}, \quad m_{p}>0, \quad m_{p} \subset \Re .
$$

We choose the orthonormal eigenvector basis at leading order to be given by [29] ${ }^{10}$

$$
\vec{\rho}_{a}^{\star}=\frac{\vec{z}}{\|\vec{z}\|}, \quad \vec{\rho}_{b}^{\star}=\frac{\vec{z}^{\star} \times(\vec{y} \times \vec{z})}{\|\vec{z}\|\|\vec{z} \times \vec{y}\|}, \quad \vec{\rho}_{c}^{\star}=\frac{\vec{y}^{\star} \times \vec{z}^{\star}}{\|\vec{z} \times \vec{y}\|} .
$$

With this convention choice, the mass of the heaviest neutrino is given by $m_{a}=|\vec{z}|^{2} v^{2} / 2 m_{3}$ at leading order and without loss of generality. The lighter neutrinos are introduced as perturbations [29]. This can also be done without loss of generality. If the FSE used to introduce these effects is a convergent expansion with small higher order terms depends upon the UV parameters in the seesaw model. The perturbations to the eigenvectors and eigenvalues are given in ref. [29]. We also define the eigenvectors $U(\nu, L)=\left(\vec{v}_{c}^{\star}, \vec{v}_{b}^{\star}, \vec{v}_{a}^{\star}\right)$ which include the perturbations of the eigenvectors to obtain the full complex mass matrix at dimension five in the SMEFT. Note that all eigenvectors in this discussion are normalized to ensure unitarity of the PMNS matrix order by order in the FSE. ${ }^{11}$ The PNMS matrix is defined in direct analogy to the CKM matrix as

$$
\mathcal{U}_{\mathrm{PMNS}}=\mathcal{U}^{\dagger}(e, L) \mathcal{U}(\nu, L) .
$$

The rotation matrix $\mathcal{U}(e, L)$ is introduced to diagonalize the lepton mass matrix

$$
\mathcal{M}_{e}=v Y_{e} / \sqrt{2}, \quad \mathcal{U}(e, L)^{\dagger} \mathcal{M}_{e}^{\dagger} \mathcal{M}_{e} \mathcal{U}(e, L)=\operatorname{diag}\left\{m_{e}^{2}, m_{\mu}^{2}, m_{\tau}^{2}\right\}
$$

Defining the orthonormal (column) eigenvectors of the lepton rotation matrix $\mathcal{U}^{\dagger}(e, L)$ as $\vec{\sigma}_{i}$ with $\mathcal{U}^{\dagger}(e, L)=\left(\vec{\sigma}_{1}^{\star}, \vec{\sigma}_{2}^{\star}, \vec{\sigma}_{3}^{\star}\right)^{T}$ we have

$$
\mathcal{U}_{\mathrm{PNMS}}^{\text {eigen }}=\left(\begin{array}{l}
\vec{v}_{c} \cdot \vec{\sigma}_{1}^{\star} \vec{v}_{b} \cdot \vec{\sigma}_{1}^{\star} \vec{v}_{a} \cdot \vec{\sigma}_{1}^{\star} \\
\vec{v}_{c} \cdot \vec{\sigma}_{2}^{\star} \vec{v}_{b} \cdot \vec{\sigma}_{2}^{\star} \vec{v}_{a} \cdot \vec{\sigma}_{2}^{\star} \\
\vec{v}_{c} \cdot \vec{\sigma}_{3}^{\star} \vec{v}_{b} \cdot \vec{\sigma}_{3}^{\star} \vec{v}_{a} \cdot \vec{\sigma}_{3}^{\star}
\end{array}\right)
$$

As we are assuming Majorana neutrino masses in a seesaw model, this matrix can be compared to the standard parameterization for unitary matricies. Define

$$
P\left(c_{1}, s_{1}, c_{2}, s_{2}, c_{3}, s_{3}, \theta\right)=\left(\begin{array}{ccc}
c_{1} c_{3} & s_{1} c_{3} & s_{3} e^{-i \theta} \\
-s_{1} c_{2}-c_{1} s_{2} s_{3} e^{i \theta} & c_{1} c_{2}-s_{1} s_{3} s_{2} e^{i \theta} & s_{2} c_{3} \\
s_{1} s_{2}-c_{1} c_{2} s_{3} e^{i \theta} & -c_{1} s_{2}-s_{1} c_{2} s_{3} e^{i \theta} & c_{2} c_{3}
\end{array}\right),
$$

and

$$
\Theta\left(v_{1}, v_{2}, v_{3}\right)=\left(\begin{array}{ccc}
e^{i v_{1}} & 0 & 0 \\
0 & e^{i v_{2}} & 0 \\
0 & 0 & e^{i v_{3}}
\end{array}\right)
$$

\footnotetext{
${ }^{10}$ See the appendix for details on the dot and cross products in the flavour space defined over the field $\mathbb{C}^{3}$.

${ }^{11}$ If the PMNS matrix is not unitary, this corresponds to the FSE not converging.
} 
so that

$$
\mathcal{U}_{\mathrm{PNMS}}^{s_{i j}}=P\left(c_{12}, s_{12}, c_{23}, s_{23}, c_{13}, s_{13}, \delta\right) \Theta\left(0, \alpha_{21} / 2, \alpha_{31} / 2\right),
$$

with the convention choice $c_{i j}=\cos \theta_{i j}, s_{i j}=\sin \theta_{i j}$ and angles $\theta_{i j}=(0, \pi / 2)$. Here $\delta=(0,2 \pi), \alpha_{21}$ and $\alpha_{31}$ are $\mathrm{CP}$ violating phases.

Each entry in eq. (5.10) is a Hermitian inner product characterized by two parameters, naively leading to eighteen parameters. Comparing to eq. (5.13) which is a general low energy parameterization in terms of six parameters (three moduli angles and three phases) makes clear that there is a redundancy of description in this naive interpretation. However, the eigenvectors sets making up the rotation matricies have to be orthogonal to lead to three masses for the charged leptons and neutrinos. As such the third vector is not independent in its flavour space orientation, although it can carry a relative phase. This leads to nine parameters in each case. Using the relation

$$
\mathcal{U}(\nu, L)=\mathcal{U}(e, L) \mathcal{U}_{\mathrm{PNMS}}^{s_{i j}},
$$

with the $\mathcal{U}(\nu, L)$ and $\mathcal{U}(e, L)$ expanded in their eigenvectors we find the leading order result

$$
\begin{aligned}
\vec{\rho}_{c}^{\star} & =\left(s_{12} s_{23}-c_{12} c_{23} s_{13} e^{i \delta}\right) \vec{\sigma}_{3}+\left(-s_{12} c_{23}-c_{12} s_{23} s_{13} e^{i \delta}\right) \vec{\sigma}_{2}+c_{12} c_{13} \vec{\sigma}_{1}, \\
\vec{\rho}_{b}^{\star} e^{\frac{-i \alpha_{21}}{2}} & =\left(-c_{12} s_{23}-c_{23} s_{12} s_{13} e^{i \delta}\right) \vec{\sigma}_{3}+\left(c_{12} c_{23}-s_{23} s_{12} s_{13} e^{i \delta}\right) \vec{\sigma}_{2}+c_{13} s_{12} \vec{\sigma}_{1}, \\
\vec{\rho}_{a}^{\star} e^{\frac{-i \alpha_{31}}{2}} & =c_{13} c_{23} \vec{\sigma}_{3}+c_{13} s_{23} \vec{\sigma}_{2}+e^{-i \delta} s_{13} \vec{\sigma}_{1} .
\end{aligned}
$$

This expression for $\vec{\rho}_{a}^{\star}$ then defines $\vec{z} /\|\vec{z}\|$ at leading order in the FSE. Further, without loss of generality $\|\vec{z}\|=1$ at leading order. The $\vec{\sigma}_{i}$ are a set of orthonormal eigenvectors for the unitary matrix $\mathcal{U}(e, L)$. These vectors form a basis for the field $\mathbb{C}^{3}$, as they diagonalize $\mathcal{M}_{e}^{\dagger} \mathcal{M}_{e}$, a Hermitian positive matrix also defined over the field $\mathbb{C}^{3}$. We can expand the unknown complex flavour vector $y$ into this orthonormal basis. Using the orthogonality and normalization properties of the basis vectors of this space, and a general parameterization of these vectors, then allows the use of the systematic EFT expansion, without the rotation matrix $\mathcal{U}(e, L)$ being chosen to have a fixed form. We can always define a flavour vector such that

$$
\vec{y}=A^{\prime} \vec{\sigma}_{1}+B^{\prime} \vec{\sigma}_{2}+C^{\prime} \vec{\sigma}_{3},
$$

with $A^{\prime}, B^{\prime}, C^{\prime} \subset \mathbb{C}$. The vectors $\vec{\sigma}_{i}$ can be parameterized as discussed in the appendix. These vectors satisfy the complex algebra $\vec{\sigma}_{i} \times \vec{\sigma}_{j}=\epsilon_{i j k} \vec{\sigma}_{k}$ and $\vec{\sigma}_{i}^{\star} \times \vec{\sigma}_{j}^{\star}=\epsilon_{i j k} \vec{\sigma}_{k}^{\star}$ without loss of generality, and we note that the $\vec{\sigma}_{i}^{\star}$ are projectable onto $\vec{\sigma}_{i}$. Solving the general system of equations is straightforward, if tedious. As an example of the utility of this formalism we examine and falsify a simple case. We show that a UV scenario where the second heavy state integrated out couples to the SM as

$$
\vec{y}=A^{\prime} \vec{\sigma}_{1},
$$

does not satisfy eqs. (5.15)-(5.17) and eqs. (5.7) simultaneously in the limit $s_{13} \rightarrow 0 .{ }^{12}$ This simple example suffices for our purpose of demonstrating how to perturb in the unknown

\footnotetext{
${ }^{12} \mathrm{As} s_{13}^{2} \simeq 0.02$ for $\delta m>0$ or $\delta m<0$ [13] the limit considered is experimentally motivated.
} 
$\vec{\sigma}_{i}$ and still obtain physical conclusions on the possible UV theories extending the SM. It is straightforward to derive that in this limit eqs. (5.15)-(5.17) and eqs. (5.7) require

$$
\left|A^{\prime}\right|^{2}=\frac{\|\vec{z} \times \vec{y}\|^{2}}{c_{13}^{2}} .
$$

with the projection coefficients of the $\vec{\sigma}_{i}^{\star}$ vectors back onto the $\vec{\sigma}_{i}$ basis of $\mathbb{C}^{3}$ required to satisfy (for $s_{13} \rightarrow 0$ )

$$
\begin{aligned}
& \sigma_{1}^{\star} \cdot \sigma_{2}=e^{i \alpha_{21} / 2} c_{12} c_{23} \frac{\|\vec{z} \times \vec{y}\|}{\left(A^{\prime}\right)^{\star}} \\
& \sigma_{1}^{\star} \cdot \sigma_{1}=e^{i \alpha_{21} / 2} s_{12} \frac{\|\vec{z} \times \vec{y}\|}{\left(A^{\prime}\right)^{\star}} \\
& \sigma_{3}^{\star} \cdot \sigma_{3}=\frac{c_{23}^{2}}{s_{23}^{2}} \sigma_{2}^{\star} \cdot \sigma_{2}+e^{-i \alpha_{31} / 2} \frac{s_{12}}{s_{23}^{2}} \frac{\|\vec{z} \times \vec{y}\|}{A^{\prime}}-2 e^{-i \alpha_{31} / 2} \frac{c_{23}^{2}}{s_{23}^{2}} s_{12} \frac{\|\vec{z} \times \vec{y}\|}{A^{\prime}} \\
& \sigma_{3}^{\star} \cdot \sigma_{2}=\frac{c_{23}}{s_{23}} \sigma_{2}^{\star} \cdot \sigma_{2}-e^{-i \alpha_{31} / 2} \frac{c_{23}}{s_{23}} s_{12} \frac{\|\vec{z} \times \vec{y}\|}{A^{\prime}} \\
& \sigma_{3}^{\star} \cdot \sigma_{1}=\frac{c_{23}}{s_{23}} \sigma_{2}^{\star} \cdot \sigma_{1}+e^{-i \alpha_{31} / 2} \frac{c_{12}}{s_{23}} \frac{\|\vec{z} \times \vec{y}\|}{A^{\prime}}, \\
& \sigma_{2}^{\star} \cdot \sigma_{3}=\frac{c_{23} s_{23}}{c_{23}^{2}-s_{23}^{2}} \sigma_{3}^{\star} \cdot \sigma_{3}-\frac{c_{23} s_{23}}{c_{23}^{2}-s_{23}^{2}} \sigma_{2}^{\star} \cdot \sigma_{2}, \\
& \sigma_{2} \cdot \sigma_{3}^{\star}=\frac{c_{23} s_{23}}{c_{23}^{2}-s_{23}^{2}} \sigma_{3} \cdot \sigma_{3}^{\star}-\frac{c_{23} s_{23}}{c_{23}^{2}-s_{23}^{2}} \sigma_{2} \cdot \sigma_{2}^{\star} \\
& \sigma_{1}^{\star} \cdot \sigma_{3}=-\frac{s_{23}}{c_{23}} \sigma_{1}^{\star} \cdot \sigma_{2}, \\
& \sigma_{1} \cdot \sigma_{3}^{\star}=-\frac{s_{23}}{c_{23}} \sigma_{1} \cdot \sigma_{2}^{\star} .
\end{aligned}
$$

This scenario is falsified as it is not possible to simultaneously satisfy these equations using the general parameterization of the unitary matrix $\mathcal{U}(e, L)$, defining the eigenvectors $\vec{\sigma}_{i}^{\star}$ and $\vec{\sigma}_{i}$. It is easiest to see this point examining the ratio of the first two equations. The right hand side of this ratio is necessarily $\in \mathbb{R}$, while this does not hold for the left hand side for any non-zero value of $\delta^{\ell}$. Vanishing $\delta^{\ell}$ leads to the other equations not being satisfied. Note that this conclusion is unchanged if the $\beta_{i}^{\ell}$ phases of $\mathcal{U}(e, L)$ are retained or not. It follows that irrespective of the particular $\vec{\sigma}_{i}$ chosen, the flavour orientation of the seesaw scenario given in eq. (5.19) is not consistent at LO in the FSE (for $s_{13} \rightarrow 0$ ).

\section{Conclusions}

In this paper we have systematically matched the minimal seesaw scenario onto the SMEFT up to dimension seven in the operator expansion. We have reported the results on $\mathcal{L}^{(7)}$ in section 3.3. These corrections can be neglected until perturbations on the $\mathcal{Q}_{5}$ operator Wilson coefficient in the Flavour Space Expansion are comparable to a $\omega^{2} v^{2} / m_{p}^{2}$ SMEFT operator expansion correction. We have shown how the neutrino mass matrix perturbations due to higher mass dimension operators include effects introduced when integrating out the $N_{p}$ states in sequence. We have demonstrated how a consistent matching at $\mathcal{L}^{(6)}$, retaining 
the $N_{p}$ in the spectrum after the $N_{1}$ state is integrated out, is essential in avoiding matching ambiguities. We have embedded the Flavour Space Expansion in the SMEFT formalism and we have developed a novel technique to perturb in the eigenvectors of the rotation matrix $\mathcal{U}(e, L)$. By treating these vectors as a basis for $\mathbb{C}^{3}$ to expand the seesaw theory flavour vectors, one can use the FSE to obtain physical conclusions independent of the form of $\mathcal{U}(e, L)$. We stress this technique is very general and not limited to the minimal seesaw model, or using the FSE. The results of this work embed the expansions present in neutrino phenomenology into a well defined effective field theory framework.

\section{Acknowledgments}

MT acknowledges generous support from the Villum Fonden and partial support by the Danish National Research Foundation (DNRF91). We thank Yun Jiang and Ilaria Brivio for comments on the manuscript, and an anonymous reviewer for a very good question in the review process on EOM effects.

\section{A $\quad \mathbb{C}^{3}$ algebra for eigenvectors diagonalizing a mass matrix}

The dot and cross products act on vectors that have entries defined over the field $\mathbb{C}^{3}$. The dot product is defined to be a Hermitian inner product that is anti-linear in its first entry acting on these vectors so that

$$
x \cdot y=x_{i}^{\star} y^{i},
$$

with the index $i$ summed over $\{1,2,3\}$. Also note $\|x\|=\sqrt{x \cdot x}$ and the cross product is defined as $x \times y=\left((x \times y)_{\Re}\right)^{\star}$. Here we are indicating complex conjugation of the entries of the usual cross product defined for vectors, that have entries defined on the field $\Re$. The cross product definition employed here can actually be formally derived using octonion multiplication $[43,44]$, which also opens up the possibility of further group theory analysis on this approach in flavour space.

Despite the fact that the lepton rotation matrix $\mathcal{U}(e, L)$ is completely unknown, we can perturb around the eigenvectors of this unknown matrix in the FSE. The lepton masses are diagonalized in a bi-unitary transformation

$$
\mathcal{U}(e, R)^{\dagger} \mathcal{M}_{e} \mathcal{U}(e, L)=\operatorname{diag}\left\{m_{e} m_{\mu}, m_{\tau}\right\}
$$

and $\mathcal{U}(e, L)$ also acts to diagonalize $\mathcal{M}_{e}^{\dagger} \mathcal{M}_{e}$. As $\mathcal{U}(e, L)$ is a unitary matrix, we can parameterize it by the product of three unitary matricies in complete generality so that

$$
\mathcal{U}(e, L)^{T}=\Theta\left(\beta_{1}^{\ell}, \beta_{2}^{\ell}, \beta_{3}^{\ell}\right) P\left(c_{12}^{\ell}, s_{12}^{\ell}, c_{23}^{\ell}, s_{23}^{\ell}, c_{13}^{\ell}, s_{13}^{\ell}, \delta^{\ell}\right) \Theta\left(\alpha_{1}^{\ell}, \alpha_{2}^{\ell}, \alpha_{3}^{\ell}\right) .
$$


This introduces ten parameters into the parameterization of this matrix, instead of the usual nine parameters for a unitary $3 \times 3$ matrix. We use the redundancy in one phase introduced to establish the algebra of the eigenvectors we wish to perturb in. From this general parameterization we have

$$
\begin{aligned}
\frac{\sigma_{1}^{T}}{e^{i \beta_{1}^{\ell}}} & =\left\{c_{12}^{\ell} c_{13}^{\ell} e^{i \alpha_{1}^{\ell}}, c_{13}^{\ell} s_{12}^{\ell} e^{i \alpha_{2}^{\ell}}, s_{13}^{\ell} e^{i\left(\alpha_{3}^{\ell}-\delta^{\ell}\right)}\right\}, \\
\frac{\sigma_{2}^{T}}{e^{i \beta_{2}^{\ell}}} & =\left\{\left(-c_{23}^{\ell} s_{12}^{\ell}-c_{12}^{\ell} s_{13}^{\ell} s_{23}^{\ell} e^{i \delta^{\ell}}\right) e^{i \alpha_{1}^{\ell}},\left(c_{12}^{\ell} c_{23}^{\ell}-s_{12}^{\ell} s_{13}^{\ell} s_{23}^{\ell} e^{i \delta^{\ell}}\right) e^{i \alpha_{2}^{\ell}}, c_{13}^{\ell} s_{23}^{\ell} e^{i \alpha_{3}^{\ell}}\right\}, \\
\frac{\sigma_{3}^{T}}{e^{i \beta_{3}^{\ell}}} & =\left\{\left(s_{23}^{\ell} s_{12}^{\ell}-c_{12}^{\ell} s_{13}^{\ell} c_{23}^{\ell} e^{i \delta^{\ell}}\right) e^{i \alpha_{1}^{\ell}},\left(-c_{12}^{\ell} s_{23}^{\ell}-s_{12}^{\ell} s_{13}^{\ell} c_{23}^{\ell} e^{i \delta^{\ell}}\right) e^{i \alpha_{2}^{\ell}}, c_{13}^{\ell} c_{23}^{\ell} e^{i \alpha_{3}^{\ell}}\right\} .
\end{aligned}
$$

One can then directly determine the complex algebra $\vec{\sigma}_{i} \times \vec{\sigma}_{j}=\epsilon_{i j k} \vec{\sigma}_{k}$ is present when the phase convention choice $\alpha_{1}^{\ell}+\alpha_{2}^{\ell}+\alpha_{3}^{\ell}+\beta_{1}^{\ell}+\beta_{2}^{\ell}+\beta_{3}^{\ell}=2 \pi n, n \subset \mathbb{Z}$ is made. A phase choice of this form is allowed, and reduces the number of free parameters in the parameterization of the unitary matrix $\mathcal{U}(e, L)$ to nine. It follows directly that $\vec{\sigma}_{i}^{\star} \times \vec{\sigma}_{j}^{\star}=\epsilon_{i j k} \vec{\sigma}_{k}^{\star}$ in general. It is also required to know the projection coefficients of the $\vec{\sigma}_{i}^{\star}$ onto the basis of vectors $\vec{\sigma}_{i}$ to perform the eigenvector perturbations in a general way. They can be derived directly using the definition of the Hermitian inner product and recalling $\vec{\sigma}_{i}^{\star} \cdot \vec{\sigma}_{j}=\left(\vec{\sigma}_{j} \cdot \vec{\sigma}_{i}^{\star}\right)^{\star}$.

To simplify the intermediate steps of the calculation involving $\vec{\sigma}_{i}$ and $\vec{\sigma}_{i}^{\star}$ it can be convenient to re-phase the charged lepton field to make the eigenvalues of $\mathcal{M}_{e}^{\dagger} \mathcal{M}_{e}$ positive and remove the $\alpha_{i}$ from eq. (A.3) without physical effect. ${ }^{13}$ The $\beta_{i}$ phases in eq. (A.3) define a similarity transformation

$$
\mathcal{M}_{e}^{\dagger} \mathcal{M}_{e} \rightarrow \Theta\left(\beta_{1}^{\ell}, \beta_{2}^{\ell}, \beta_{3}^{\ell}\right)^{\dagger} \mathcal{M}_{e}^{\dagger} \mathcal{M}_{e} \Theta\left(\beta_{1}^{\ell}, \beta_{2}^{\ell}, \beta_{3}^{\ell}\right)
$$

One can also choose a parameterization of $\mathcal{U}(e, L)$ where these $\beta_{i}$ intermediate unphysical phases vanish. This similarity transformation leaves the eigenvalues of $\mathcal{M}_{e}^{\dagger} \mathcal{M}_{e}$ invariant but does not leave the eigenvectors invariant in general. Choosing this phase convention fixes a general class of $\vec{\sigma}_{i}$ to perturb around as a basis for $\mathbb{C}^{3}$. As physically observable effects due to $\mathcal{U}_{\text {PMNS }}$ only come about due to the relationship between the eigenvectors sets $\vec{\sigma}_{i}^{\star}$ and $\rho_{i}^{\star}$ this can be done as a convention choice.

\footnotetext{
${ }^{13}$ For related discussions see refs. [45-48].
} 


\section{B Operator basis of ref. [1]}

\begin{tabular}{|l|l|}
\hline \multicolumn{2}{|c|}{$1: \psi^{2} H^{4}+$ h.c. } \\
\hline $\mathcal{Q}_{\ell H}$ & $\epsilon_{i j} \epsilon_{m n}\left(\ell_{L}^{i} C \ell_{L}^{m}\right) H^{j} H^{n}\left(H^{\dagger} H\right)$ \\
\hline
\end{tabular}

\begin{tabular}{|l|l|}
\hline \multicolumn{2}{|c|}{$3: \psi^{2} H^{3} D+$ h.c. } \\
\hline $\mathcal{Q}_{\ell H D e}$ & $\epsilon_{i j} \epsilon_{m n}\left(\ell_{L}^{i} C \gamma_{\mu} e_{R}\right) H^{j} H^{m} D^{\mu} H^{n}$ \\
\hline
\end{tabular}

\begin{tabular}{|l|l|}
\hline \multicolumn{2}{|c|}{$2: \psi^{2} H^{2} D^{2}+$ h.c. } \\
\hline $\mathcal{Q}_{\ell H D}^{(1)}$ & $\epsilon_{i j} \epsilon_{m n} \ell_{L}^{i} C\left(D^{\mu} \ell_{L}^{j}\right) H^{m}\left(D_{\mu} H^{n}\right)$ \\
$\mathcal{Q}_{\ell H D}^{(2)}$ & $\epsilon_{i m} \epsilon_{j n} \ell_{L}^{i} C\left(D^{\mu} \ell_{L}^{j}\right) H^{m}\left(D_{\mu} H^{n}\right)$ \\
\hline
\end{tabular}

\begin{tabular}{|c|c|}
\hline \multicolumn{2}{|c|}{$4: \psi^{2} H^{2} X+$ h.c. } \\
\hline $\mathcal{Q}_{\ell H B}$ & $\epsilon_{i j} \epsilon_{m n}\left(\ell_{L}^{i} C \sigma_{\mu \nu} \ell_{L}^{m}\right) H^{j} H^{n} B^{\mu \nu}$ \\
$\mathcal{Q}_{\ell H W}$ & $\epsilon_{i j}\left(\tau^{I} \epsilon\right)_{m n}\left(\ell_{L}^{i} C \sigma_{\mu \nu} \ell_{L}^{m}\right) H^{j} H^{n} W^{I \mu \nu}$ \\
\hline
\end{tabular}

\begin{tabular}{|l|c|}
\hline \multicolumn{2}{|c|}{$5: \psi^{4} D+$ h.c. } \\
\hline $\mathcal{Q}_{\ell \ell \bar{d} u D}^{(1)}$ & $\epsilon_{i j}\left(\overline{d_{R}} \gamma_{\mu} u_{R}\right)\left(\ell_{L}^{i} C D^{\mu} \ell_{L}^{j}\right)$ \\
$\mathcal{Q}_{\ell \ell \bar{d} u D}^{(2)}$ & $\epsilon_{i j}\left(\overline{d_{R}} \gamma_{\mu} u_{R}\right)\left(\ell_{L}^{i} C \sigma^{\mu \nu} D_{\nu} \ell_{L}^{j}\right)$ \\
$\mathcal{Q}_{\overline{\ell Q d d}}^{(1)}$ & $\left(Q_{L} C \gamma_{\mu} d_{R}\right)\left(\overline{\ell_{L}} D^{\mu} d_{R}\right)$ \\
$\mathcal{Q}_{\bar{\ell} Q d d D}^{(2)}$ & $\left(\overline{\ell_{L}} \gamma_{\mu} q_{L}\right)\left(d_{R} C D^{\mu} d_{R}\right)$ \\
$\mathcal{Q}_{d d d \bar{e} D}$ & $\left(\overline{e_{R}} \gamma_{\mu} d_{R}\right)\left(d_{R} C D^{\mu} d_{R}\right)$ \\
\hline
\end{tabular}

\begin{tabular}{|c|c|}
\hline \multicolumn{2}{|c|}{$6: \psi^{4} H+$ h.c. } \\
\hline $\mathcal{Q}_{\ell \ell \ell \bar{e} H}$ & $\epsilon_{i j} \epsilon_{m n}\left(\overline{e_{R}} \ell_{L}^{i}\right)\left(\ell_{L}^{j} C \ell_{L}^{m}\right) H^{n}$ \\
$\mathcal{Q}_{\ell \ell Q \bar{d} H}^{(1)}$ & $\epsilon_{i j} \epsilon_{m n}\left(\overline{d_{R}} \ell_{L}^{i}\right)\left(q_{L}^{j} C \ell_{L}^{m}\right) H^{n}$ \\
$\mathcal{Q}_{\ell \ell Q \bar{d} H}^{(2)}$ & $\epsilon_{i m} \epsilon_{j n}\left(\overline{d_{R}} \ell_{L}^{i}\right)\left(q_{L}^{j} C \ell_{L}^{m}\right) H^{n}$ \\
$\mathcal{Q}_{\ell \ell \bar{Q} u H}$ & $\epsilon_{i j}\left(\overline{q_{L_{m}}} u_{R}\right)\left(\ell_{L}^{m} C \ell_{L}^{i}\right) H^{j}$ \\
$\mathcal{Q}_{\bar{\ell} Q Q d H}$ & $\epsilon_{i j}\left(\overline{\ell_{L_{m}}} d_{R}\right)\left(q_{L}^{m} C q_{L}^{i}\right) \tilde{H}^{j}$ \\
$\mathcal{Q}_{\bar{\ell} d d d H}$ & $\left(d_{R} C d_{R}\right)\left(\overline{\ell_{L}} d_{R}\right) H$ \\
$\mathcal{Q}_{\bar{\ell} u d d H}$ & $\left(\overline{\ell_{L}} d_{R}\right)\left(u_{R} C d_{R}\right) \tilde{H}$ \\
$\mathcal{Q}_{\ell e u \bar{d} H}$ & $\epsilon_{i j}\left(\ell_{L}^{i} C \gamma_{\mu} e_{R}\right)\left(\overline{d_{R}} \gamma^{\mu} u_{R}\right) H^{j}$ \\
$\mathcal{Q}_{\bar{e} Q d d H}$ & $\epsilon_{i j}\left(\overline{e_{R}} Q_{L}^{i}\right)\left(d_{R} C d_{R}\right) \tilde{H}^{j}$ \\
\hline
\end{tabular}

Table 1. The operator basis of ref. [1] matched onto in this work. Here the spinors are in four component notation and $C=-i \gamma_{2} \gamma_{0}$ in the chiral basis we employ.

Open Access. This article is distributed under the terms of the Creative Commons Attribution License (CC-BY 4.0), which permits any use, distribution and reproduction in any medium, provided the original author(s) and source are credited.

\section{References}

[1] L. Lehman, Extending the Standard Model Effective Field Theory with the Complete Set of Dimension-7 Operators, Phys. Rev. D 90 (2014) 125023 [arXiv:1410.4193] [INSPIRE].

[2] S. Weinberg, Baryon and Lepton Nonconserving Processes, Phys. Rev. Lett. 43 (1979) 1566 [INSPIRE].

[3] F. Wilczek and A. Zee, Operator Analysis of Nucleon Decay, Phys. Rev. Lett. 43 (1979) 1571 [INSPIRE].

[4] W. Buchmüller and D. Wyler, Effective Lagrangian Analysis of New Interactions and Flavor Conservation, Nucl. Phys. B 268 (1986) 621 [INSPIRE]. 
[5] B. Grzadkowski, M. Iskrzynski, M. Misiak and J. Rosiek, Dimension-Six Terms in the Standard Model Lagrangian, JHEP 10 (2010) 085 [arXiv: 1008.4884] [INSPIRE].

[6] L.F. Abbott and M.B. Wise, The Effective Hamiltonian for Nucleon Decay, Phys. Rev. D 22 (1980) 2208 [inSPIRE].

[7] L. Lehman and A. Martin, Hilbert Series for Constructing Lagrangians: expanding the phenomenologist's toolbox, Phys. Rev. D 91 (2015) 105014 [arXiv:1503.07537] [INSPIRE].

[8] L. Lehman and A. Martin, Low-derivative operators of the Standard Model effective field theory via Hilbert series methods, JHEP 02 (2016) 081 [arXiv:1510.00372] [INSPIRE].

[9] B. Henning, X. Lu, T. Melia and H. Murayama, 2, 84, 30, 993, 560, 15456, 11962, 261485, ...: higher dimension operators in the SM EFT, JHEP 08 (2017) 016 [arXiv:1512.03433] [INSPIRE].

[10] S. Willenbrock, Symmetries of the standard model, hep-ph/0410370 [INSPIRE].

[11] A. de Gouvêa, J. Herrero-Garcia and A. Kobach, Neutrino Masses, Grand Unification and Baryon Number Violation, Phys. Rev. D 90 (2014) 016011 [arXiv:1404.4057] [INSPIRE].

[12] A. Kobach, Baryon Number, Lepton Number and Operator Dimension in the Standard Model, Phys. Lett. B 758 (2016) 455 [arXiv:1604.05726] [INSPIRE].

[13] Particle Data Group collaboration, C. Patrignani et al., Review of Particle Physics, Chin. Phys. C 40 (2016) 100001.

[14] P. Minkowski, $\mu \rightarrow$ e $\gamma$ at a Rate of One Out of $10^{9}$ Muon Decays?, Phys. Lett. 67B (1977) 421 [INSPIRE].

[15] M. Gell-Mann, P. Ramond and R. Slansky, Complex Spinors and Unified Theories, Conf. Proc. C 790927 (1979) 315 [arXiv:1306.4669] [inSPIRE].

[16] T. Yanagida, Horizontal symmetry and masses of neutrinos, Conf. Proc. C 7902131 (1979) 95 [INSPIRE].

[17] R.N. Mohapatra and G. Senjanović, Neutrino Mass and Spontaneous Parity Violation, Phys. Rev. Lett. 44 (1980) 912 [inSPIRE].

[18] A. Broncano, M.B. Gavela and E.E. Jenkins, The Effective Lagrangian for the seesaw model of neutrino mass and leptogenesis, Phys. Lett. B 552 (2003) 177 [Erratum ibid. B 636 (2006) 332] [hep-ph/0210271] [INSPIRE].

[19] M.B. Gavela, T. Hambye, D. Hernandez and P. Hernández, Minimal Flavour Seesaw Models, JHEP 09 (2009) 038 [arXiv:0906.1461] [INSPIRE].

[20] M.B. Gavela, D. Hernandez, T. Ota and W. Winter, Large gauge invariant non-standard neutrino interactions, Phys. Rev. D 79 (2009) 013007 [arXiv:0809.3451] [INSPIRE].

[21] A. Abada, C. Biggio, F. Bonnet, M.B. Gavela and T. Hambye, Low energy effects of neutrino masses, JHEP 12 (2007) 061 [arXiv:0707.4058] [INSPIRE].

[22] A. Broncano, M.B. Gavela and E.E. Jenkins, Neutrino physics in the seesaw model, Nucl. Phys. B 672 (2003) 163 [hep-ph/0307058] [INSPIRE].

[23] F. Bonnet, M. Hirsch, T. Ota and W. Winter, Systematic study of the D $=5$ Weinberg operator at one-loop order, JHEP 07 (2012) 153 [arXiv:1204.5862] [INSPIRE].

[24] F. Bonnet, D. Hernandez, T. Ota and W. Winter, Neutrino masses from higher than $D=5$ effective operators, JHEP 10 (2009) 076 [arXiv:0907.3143] [INSPIRE]. 
[25] F. del Aguila, S. Bar-Shalom, A. Soni and J. Wudka, Heavy Majorana Neutrinos in the Effective Lagrangian Description: Application to Hadron Colliders, Phys. Lett. B 670 (2009) 399 [arXiv: 0806.0876] [INSPIRE].

[26] F. del Aguila, A. Aparici, S. Bhattacharya, A. Santamaria and J. Wudka, Effective Lagrangian approach to neutrinoless double beta decay and neutrino masses, JHEP 06 (2012) 146 [arXiv:1204.5986] [INSPIRE].

[27] S. Bhattacharya and J. Wudka, Dimension-seven operators in the standard model with right handed neutrinos, Phys. Rev. D 94 (2016) 055022 [Erratum ibid. D 95 (2017) 039904] [arXiv: 1505.05264] [INSPIRE].

[28] P.W. Angel, N.L. Rodd and R.R. Volkas, Origin of neutrino masses at the LHC: $\Delta L=2$ effective operators and their ultraviolet completions, Phys. Rev. D 87 (2013) 073007 [arXiv: 1212.6111] [INSPIRE].

[29] B. Grinstein and M. Trott, An Expansion for Neutrino Phenomenology, JHEP 09 (2012) 005 [arXiv: 1203.4410] [INSPIRE].

[30] B. Pontecorvo, Mesonium and anti-mesonium, Sov. Phys. JETP 6 (1957) 429 [Zh. Eksp. Teor. Fiz. 33 (1957) 549] [INSPIRE].

[31] Z. Maki, M. Nakagawa and S. Sakata, Remarks on the unified model of elementary particles, Prog. Theor. Phys. 28 (1962) 870 [InSPIRE].

[32] E. Majorana, Teoria simmetrica dell'elettrone e del positrone, Nuovo Cim. 14 (1937) 171 [INSPIRE].

[33] Y. Liao and X.-D. Ma, Renormalization Group Evolution of Dimension-seven Baryon- and Lepton-number-violating Operators, JHEP 11 (2016) 043 [arXiv: 1607.07309] [INSPIRE].

[34] Y. Liao and X.-D. Ma, Operators up to Dimension Seven in Standard Model Effective Field Theory Extended with Sterile Neutrinos, Phys. Rev. D 96 (2017) 015012 [arXiv: 1612.04527] [INSPIRE].

[35] A. Broncano, M.B. Gavela and E.E. Jenkins, Renormalization of lepton mixing for Majorana neutrinos, Nucl. Phys. B 705 (2005) 269 [hep-ph/0406019] [INSPIRE].

[36] E.E. Jenkins, A.V. Manohar and M. Trott, On Gauge Invariance and Minimal Coupling, JHEP 09 (2013) 063 [arXiv:1305.0017] [InSPIRE].

[37] E.E. Jenkins, A.V. Manohar and M. Trott, Renormalization Group Evolution of the Standard Model Dimension Six Operators I: Formalism and lambda Dependence, JHEP 10 (2013) 087 [arXiv: 1308.2627] [INSPIRE].

[38] H.K. Dreiner, H.E. Haber and S.P. Martin, Two-component spinor techniques and Feynman rules for quantum field theory and supersymmetry, Phys. Rept. 494 (2010) 1 [arXiv: 0812.1594] [INSPIRE].

[39] S.F. King, Atmospheric and solar neutrinos with a heavy singlet, Phys. Lett. B 439 (1998) 350 [hep-ph/9806440] [INSPIRE].

[40] S.F. King, Atmospheric and solar neutrinos from single right-handed neutrino dominance and U(1) family symmetry, Nucl. Phys. B 562 (1999) 57 [hep-ph/9904210] [InSPIRE].

[41] S.F. King, Large mixing angle $M S W$ and atmospheric neutrinos from single right-handed neutrino dominance and U(1) family symmetry, Nucl. Phys. B 576 (2000) 85 [hep-ph/9912492] [INSPIRE]. 
[42] S.F. King, Constructing the large mixing angle MNS matrix in seesaw models with right-handed neutrino dominance, JHEP 09 (2002) 011 [hep-ph/0204360] [INSPIRE].

[43] A. Cayley, Xxviii. on Jacobi's elliptic functions, in reply to the rev. brice bronwin; and on quaternions, Philos. Mag. Ser. 326 (1845) 208.

[44] J.C. Baez, The Octonions, Bull. Am. Math. Soc. 39 (2002) 145 [math/0105155] [InSPIRE].

[45] E.E. Jenkins and A.V. Manohar, Rephasing Invariants of Quark and Lepton Mixing Matrices, Nucl. Phys. B 792 (2008) 187 [arXiv:0706.4313] [InSPIRE].

[46] E.E. Jenkins and A.V. Manohar, Algebraic Structure of Lepton and Quark Flavor Invariants and CP-violation, JHEP 10 (2009) 094 [arXiv:0907.4763] [INSPIRE].

[47] C. Jarlskog, A Basis Independent Formulation of the Connection Between Quark Mass Matrices, CP-violation and Experiment, Z. Phys. C 29 (1985) 491 [inSPIRE].

[48] A. Kusenko and R. Shrock, General determination of phases in leptonic mass matrices, Phys. Lett. B 323 (1994) 18 [hep-ph/9311307] [INSPIRE]. 